\title{
Nanoliposomal irinotecan with fluorouracil for the treatment of advanced pancreatic cancer, a single institution experience
}

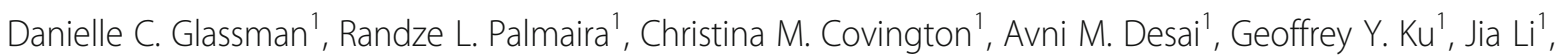
James J. Harding ${ }^{1}$, Anna M. Varghese ${ }^{1}$, Eileen M. O'Reilly ${ }^{1}$ and Kenneth H. Yu ${ }^{1,2^{*}}$ (i)

\begin{abstract}
Background: Effective treatment options for advanced pancreatic cancer are finite. NAPOLI-1, a phase III randomized trial, demonstrated the efficacy of nanoliposomal irinotecan with fluorouraci//leucovorin (nal-IRI + 5-FU/LV) for the treatment of advanced pancreatic cancer following progression on gemcitabine-based chemotherapy. There are limited additional data on the safety and efficacy of nal-IRI + 5-FU/LV following FDA approval in October 2015. We examined the post-approval safety and effectiveness of nal-IRI +5-FU/LV in advanced pancreatic cancer patients receiving treatment at Memorial Sloan Kettering Cancer Center.
\end{abstract}

Methods: A retrospective chart review was conducted of all patients beginning treatment with nal-|RI + 5-FU/LV from October 2015 through June 2017. Using the electronic medical record and institutional database, information was extracted pertaining to demographics, performance status (ECOG), prior therapies, dose, duration of treatment, adverse events, progression free survival (PFS), overall survival (OS) and treatment response.

Results: Fifty six patients were identified. Median progression free survival (PFS) was 2.9 months and median overall survival (OS) was 5.3 months. Patients with prior disease progression on irinotecan experienced PFS and OS of 2.2 and 3.9 mo, respectively. Patients without prior irinotecan exposure experienced significantly longer PFS (4.8 mo, $p=0.02)$ and OS (7.7 mo, $p=0.002)$, as did patients who received prior irinotecan without disease progression (PFS, $5.7 \mathrm{mo}$, $p=0.04 ; \mathrm{OS}, 9.0 \mathrm{mo}, p=.04)$. Progression on prior irinotecan was associated with greater lines of prior advanced disease chemotherapy (2 vs 1). Dose reductions (DR) were most frequently due to fatigue (42\%) and diarrhea (37\%), but were not associated with worse outcomes. In fact, patients with $\geq 1$ DR experienced longer PFS (5.4 $\vee 2.6$ mo, $p=0.035)$. Sequential therapy with nab-paclitaxel + gemcitabine (nab-P + Gem) followed by nal-IRI + 5-FU/LV $(n=25)$ resulted in OS of 23.0 mo. Mutations in TP53 were associated with shorter PFS.

Conclusions: These data support the safety and efficacy of nal-IRI + 5-FU/LV, reinforcing results of NAPOLI-1. Patients without disease progression on prior irinotecan fared significantly better than patients with progression, when treated with nal-IRI + 5-FU/LV. Sequential therapy with nab-P + Gem followed by nal-IRI + 5-FU/LV demonstrates encouraging median OS. These findings provide guidance for patients most likely to benefit from nal-IRI +5-FU/LV.

Keywords: Pancreatic cancer, Nanoliposomal irinotecan, MM-398, Nal-IRI, 5-fluorouracil

\footnotetext{
* Correspondence: yuk1@mskcc.org

${ }^{1}$ David M. Rubenstein Center for Pancreatic Cancer Research, Memorial Sloan

Kettering Cancer Center, Weil Cornell Medical College, New York, NY, USA

${ }^{2}$ Gastrointestinal Oncology Service, Memorial Sloan Kettering Cancer Center,

300 East 66th Street, New York, NY 10065, USA
}

(c) The Author(s). 2018 Open Access This article is distributed under the terms of the Creative Commons Attribution 4.0 International License (http://creativecommons.org/licenses/by/4.0/), which permits unrestricted use, distribution, and reproduction in any medium, provided you give appropriate credit to the original author(s) and the source, provide a link to the Creative Commons license, and indicate if changes were made. The Creative Commons Public Domain Dedication waiver (http://creativecommons.org/publicdomain/zero/1.0/) applies to the data made available in this article, unless otherwise stated. 


\section{Background}

Pancreatic ductal adenocarcinoma (PDAC) remains an intractable illness due to late stage of presentation, a propensity to metastasize, relative resistance to cytotoxic treatment and the lack of effective targeted agents. In 2017, an estimated 53,670 new cases of pancreatic cancer were diagnosed [1]. The majority of patients have either regional (11.5\%) or distant (52\%) spread at presentation. With a low 5-year survival rate of only $8.2 \%$, PDAC ranks as the 3rd leading cause of cancer deaths, with an estimated 43,090 patient deaths in 2017. It is estimated that PDAC will rise to the second leading cause of cancer mortality by 2030 [2].

The treatment landscape for advanced PDAC has significantly changed since 2010. Randomized phase III trials have demonstrated significant survival benefits of FOLFIRINOX (folinic acid, 5-fluorouracil, irinotecan and oxaliplatin) [3] or nab-paclitaxel + gemcitabine (nab-P + Gem) [4] compared with the prior standard of care, single agent gemcitabine, for frontline treatment. Nanoliposomal irinotecan (nal-IRI) is a novel formulation of irinotecan, encapsulating drug molecules within long-circulating liposome-based nanoparticles with resulting favorable pharmacokinetic and biodistribution properties [5]. Recently, the randomized phase III NAPOLI-1 trial demonstrated significant survival benefit of nal-IRI with fluorouracil/leucovorin (nal-IRI + 5-FU/LV) compared with 5-FU alone after disease-progression on gemcitabine-based chemotherapy, progression-free survival (PFS) of 3.1 vs 1.5 months, respectively $(p=0.0001)$ and overall survival (OS) of 6.1 vs 4.2 months $(p=0.012)$. Nal-IRI received FDA approval on October 22nd, 2015.

Due to the aggressiveness of this disease, and, until recently, the dearth of effective therapies, the majority of patients receive only a single line of chemotherapy [6, 7]. With the current availability of several lines of active combination therapy, studies describing outcomes of sequential therapy are greatly needed. In particular, evidence for how to best integrate nal-IRI + 5-FU/LV into the treatment algorithm is needed and to understand the dosing schedule of the regimen. This retrospective, single institution analysis was conducted to address these questions.

\section{Methods}

\section{Patients}

A retrospective review was conducted of all consecutive patients with advanced PDAC who began receiving treatment with nal-IRI + 5-FU/LV at Memorial Sloan Kettering Cancer Center (MSKCC) and its regional care network between October 2015 and June 2017. The electronic medical record (EMR) was interrogated for patient demographics, performance status (ECOG), date of diagnosis, date of advanced disease diagnosis and carbohydrate antigen 19-9 (CA 19-9) level at baseline, tumor and germline genomic results, prior treatment details and duration, nal-IRI + 5-FU/LV starting dose, nal-IRI + 5-FU/LV dose reductions, nal-IRI + 5-FU/LV treatment duration, adverse events and survival. Tumor and germline genomics were performed under an IRB approved protocol (NCT01775072). The MSK-IMPACT somatic analysis utilizes targeted next generation sequencing (NGS) of pancreatic tumor tissue to interrogate a panel of 410-481 genes. Germline analysis on DNA obtained from normal peripheral blood utilizes NGS to interrogate a panel of 76 genes associated with hereditary cancer predisposition. This retrospective analysis was granted a research waiver by the MSKCC Institutional Review Board.

\section{Outcome measures}

All treatment related adverse events (AEs) that occurred while patients were treated with nal-IRI + 5-FU/LV were collected. All AEs and SAEs were graded per National Cancer Institute Common Terminology Criteria for Adverse Events (NCI-CTCAE V4.0).

Patients were assessed every 8-12 weeks by computed tomography (CT). Disease response was assessed using RECIST version 1.1 criteria. Response by change in CA 19-9 level was recorded. Date of disease progression on nal-IRI + 5-FU/LV treatment and date of death were recorded.

\section{Statistical analysis}

Descriptive statistics were calculated as mean, median or percentages as appropriate. PFS was calculated from the time of first nal-IRI + 5-FU/LV administration to disease progression or death, whichever occurred first. Nal-IRI +5 -FU/LV OS was calculated from time of first nal-IRI +5 -FU/LV administration to death. Advanced disease OS was calculated from time of advanced disease diagnosis to death.

Patients without progression or death were censored at the last follow-up date as of November 2nd, 2017. Survival curves and median survival were estimated using the Kaplan-Meier method. Survival curves were compared using Log-rank (Mantel-Cox) test or Log-rank test for trend. Hazard ratios were calculated using Log-rank test with 95\% confidence intervals.

\section{Results}

\section{Patient and tumor characteristics}

During the accrual period, $N=56$ patients with advanced PDAC received treatment with nal-IRI + 5-FU/LV at MSKCC. All patients who received one or more administrations of nal-IRI + 5-FU/LV were included in the analysis. The patient characteristics are listed in Table 1 . The median age was 68 years, range 42 to 88 years. The significant majority of patients had metastatic disease at the time of treatment onset, with only two patients with locally advanced 
Table 1 Characteristics of patients and tumors

\begin{tabular}{|c|c|}
\hline & $N=56(\%)$ \\
\hline Median Age (years, range) & $68(42-88)$ \\
\hline \multicolumn{2}{|l|}{ Gender } \\
\hline Male & $29(52)$ \\
\hline Female & $27(48)$ \\
\hline \multicolumn{2}{|l|}{ ECOG Performance Status } \\
\hline 0 & $3(5)$ \\
\hline 1 & $41(73)$ \\
\hline 2 & $12(21)$ \\
\hline \multicolumn{2}{|l|}{ Primary tumor location } \\
\hline Head & $28(50)$ \\
\hline Body & $11(20)$ \\
\hline Tail & $12(21)$ \\
\hline Body and tail & $5(9)$ \\
\hline \multicolumn{2}{|l|}{ Stage at start of treatment } \\
\hline III & $2(4)$ \\
\hline IV & $54(96)$ \\
\hline \multicolumn{2}{|l|}{ Metastatic sites } \\
\hline Liver & $41(73)$ \\
\hline Peritoneum & $16(29)$ \\
\hline Lung & $15(27)$ \\
\hline Distant lymph nodes & $18(32)$ \\
\hline Other & $10(18)$ \\
\hline \multicolumn{2}{|l|}{ Number of metastatic sites } \\
\hline 1 & $29(52)$ \\
\hline 2 & $8(14)$ \\
\hline 3 or more & $16(29)$ \\
\hline \multicolumn{2}{|c|}{ Prior lines of advanced disease therapy } \\
\hline 0 & $4(7)$ \\
\hline 1 & $20(36)$ \\
\hline 2 & $21(38)$ \\
\hline 3 or more & $11(20)$ \\
\hline
\end{tabular}

disease. The majority (79\%) of patients had an Eastern Cooperative Oncology Group (ECOG) performance status of 0 or 1, the remainder (20\%) had an ECOG performance status of 2 . Nineteen patients had prior surgery and nine patients received prior radiation therapy. Although the majority of patients received at least one $(36 \%)$ or more $(58 \%)$ lines of chemotherapy prior to receiving nal-IRI + 5-FU/LV, 4 (7\%) patients were treated with nal-IRI + 5-FU/LV in the frontline, metastatic setting after failure of gemcitabinebased chemotherapy in the adjuvant setting.

\section{Dosing and drug delivery}

The majority of patients (70\%) started nal-IRI + 5-FU/LV treatment with a dose of nal-IRI below the recommended $70 \mathrm{mg} / \mathrm{m}^{2}$ dose level, see Table 2. The median starting dose was $55 \mathrm{mg} / \mathrm{m}^{2}$. The choice of a lower starting dose at our institution is based on physician preference. Line of therapy and ECOG performance status were not factors associated with lower starting dose. The only statistically significant factor identified was age; the median age of patients starting at full dose was 63 versus $70(p=0.01)$. The majority of patients never experienced a dose reduction of nal-IRI, with 15 (27\%) experiencing a single dose reduction and only 3 (5\%) experiencing two dose reductions. Examining the sequence of chemotherapy regimens prior to nal-IRI + 5-FU/LV treatment, the vast majority followed one of two patterns. The most common sequence was treatment with either 5-FU based chemotherapy, typically FOLFIRINOX or FOLFOX, followed by gemcitabine-based chemotherapy, typically single agent gemcitabine or nab-P + Gem, or the inverse (referred to going forward as Sequence 1). Twenty-six (46\%) experienced this pattern of treatment, followed by nal-IRI $+5-\mathrm{FU} / \mathrm{LV}$ treatment in the $3 \mathrm{rd}$ line or later. The second most common sequence, received by 25 (45\%) patients, was treatment with gemcitabine-based chemotherapy, typically either gemcitabine alone or nab-P + Gem in the frontline or adjuvant setting followed by nal-IRI + 5-FU/LV treatment in the 2nd line (referred to going forward as Sequence 2). A small number of patients, 3 (5\%), received nab-P + Gem followed by Gem/capecitabine (Cap), followed by nal-IRI + 5-FU/LV in the 3rd line. Two (4\%) patients received sequential treatment that did not fit any of these patterns due to participation in clinical trials.

Table 2 Dosing, dose reductions and sequencing of nal-IRI + 5-FU/LV $N=56(\%)$

Starting nal-IRI dose $(\mathrm{mg} / \mathrm{m} 2)$

$\begin{array}{ll}<50 & 23(41) \\ 55 & 9(16) \\ 60 & 7(13) \\ 70 & 17(30)\end{array}$

Dose reductions (\#)

$38(68)$

$15(27)$

$3(5)$

Treatment sequencing

$\begin{array}{ll}\text { FOLF }(I R I N) \text { OX } \leftarrow \rightarrow(\text { nab-P })+\text { Gem } \rightarrow \text { nal-IRI }+5 \text {-FU/LV } & 26(46) \\ \text { nab-P }+ \text { Gem } \rightarrow \text { nal-IRI }+5 \text {-FU/LV } & 25(45) \\ \text { nab-P }+ \text { Gem } \rightarrow \text { Gem/Cap } \rightarrow \text { nal-IRI + 5-FU/LV } & 3(5) \\ \text { other } & 2(4)\end{array}$




\section{Efficacy}

For the entire cohort of $N=56$ the median PFS was 2.9 months and the median OS was 5.3 months (Table 3). Three patients had a PR (5\%) and $23(41 \%)$ had SD per RECIST. Ten patients (18\%) experienced $>50 \%$ reduction of CA 19-9 at maximal response compared to baseline. Patients were classified based on whether they received irinotecan $(N=33,59 \%)$ in prior lines of chemotherapy, or not $(N=23,41 \%)$. Of patients receiving prior irinotecan, patients were further divided into those whose disease progressed on prior irinotecan-based chemotherapy $(N=27,48 \%)$, or not $(N=6,11 \%)$. The latter generally were patients who presented initially with locally advanced disease who completed a course of FOLFIRINOX chemotherapy in the neoadjuvant setting without disease progression before moving on to surgery or radiation therapy. By contrast, patients whose disease progressed on prior irinotecan-based chemotherapy typically received FOLFIRINOX as front-line therapy for metastatic disease. Patients receiving nal-IRI + 5-FU/LV after progressing on prior irinotecan-based chemotherapy experienced significantly shorter PFS and OS compared with patients not previously treated with irinotecan (PFS, $2.2 \mathrm{v}$ $4.6 \mathrm{mo}, p=0.022$; OS, 3.9 v $7.7 \mathrm{mo}, p=0.0021$ ), and also when compared with patients previously treated with irinotecan without progression (PFS, 2.2 v $5.7 \mathrm{mo}, p=0.041$; OS, 3.9 v $9.0 \mathrm{mo}, p=0.035$ ) (Fig. 1). Importantly, patients

Table 3 Overall efficacy and response to treatment with nal-IRI $+5-\mathrm{FU} / \mathrm{LV}$

\begin{tabular}{ll}
\hline & $N=56(\%)$ \\
\hline PFS (median, mo) & 2.9 \\
OS (median, mo) & 5.3 \\
Response rate & \\
$\quad$ Partial response & $3(5)$ \\
Stable disease & $23(41)$ \\
Progressive disease & $23(41)$ \\
Not evaluabe & $7(13)$ \\
CA 19-9 response (maximal response/baseline) & \\
$>1$ & $19(34)$ \\
0.5 to 1 & $15(27)$ \\
$<0.5$ & $10(18)$ \\
not evaluable & $10(18)$ \\
not measurable & $2(4)$ \\
Advanced disease, OS (median, mo) & \\
All & 24.2 \\
Sequence 1 & 25.5 \\
Sequence 2 & 23.0 \\
nab-P + Gem $\rightarrow$ Gem/Cap $\rightarrow$ nal-IRI + 5-FU/LV & 28.6 \\
other & 23.0 \\
\hline
\end{tabular}

with progression on prior irinotecan-based chemotherapy typically received nal-IRI +5-FU/LV in a later line of therapy (median, 3rd-line) compared with the other sub-groups. Looking specifically at line of advanced disease therapy, there was a significant trend to longer PFS $(p=0.0031)$ and OS $(p=0.0002)$ for patients receiving nal-IRI + 5-FU/LV in earlier lines of therapy, compared with later (Fig. 2).

ECOG performance status at start of nal-IRI + 5-FU/LV treatment was not significantly associated with PFS or OS. Twenty percent of patients in our cohort began treatment with an ECOG performance status of 2. This contrasts with patients treated with nal-IRI + 5-FU/LV in the NAPOLI-1 trial, where only $8.5 \%$ of patients began treatment with equivalent Karnofsky performance status of 70 or worse [8]. Starting dose of nal-IRI was also not significantly associated with survival, however, dose-reduction of nal-IRI was. Increasing numbers of dose reductions were associated with increased PFS $(p=0.016)$. There was also a trend to increased OS, though this did not meet statistical significance $(p=0.073)$. Comparing patients with or without any dose reductions, PFS was 5.4 v $2.6 \mathrm{mo}$ $(p=0.035)$, OS was $7.1 \times 4.5 \mathrm{mo}$ (not significant, $p=0.1226$ ).

Treatment sequences were significantly associated with survival (Fig. 3). Patients receiving Sequence 1 experienced significantly shorter PFS ( $2.2 \mathrm{v} 4.8 \mathrm{mo}, p=0.0094)$ and OS (4.1 v $9.0 \mathrm{mo}, p=0.0006)$ compared with Sequence 2. OS from the time of advanced disease diagnosis was analyzed. Median OS from time of documentation of stage III or IV disease was $24.2 \mathrm{mo}$ for all patients receiving nal-IRI + 5-FU/LV. OS was similar across all sequences of treatment (Table 3). A sequence of particular interest was Sequence 2, with patients receiving frontline Gem with or without nab-P, followed by nal-IRI + 5-FU/LV. Median OS was 23.0 mo.

\section{Safety}

Patients were evaluated for toxicity through history and physical exam, complete blood count, and comprehensive metabolic panel. Treatment was discontinued at the discretion of treating physician due to toxicity or progression of disease. The number of dose reductions and attributed reasons for dose reductions are detailed in Table 4. Of the 20 total dose reductions, the most common reasons were for fatigue and diarrhea. Some dose reductions were attributed to multiple reasons. Adverse and serious adverse events are detailed in Table 5. Compared to the pivotal NAPOLI-1 trial, overall toxicity was comparable. There were lower rates of grade 3 or 4 toxicities seen in the MSKCC patient cohort across all of the most common toxicities observed, likely due in part to the lower median starting dose administered. 


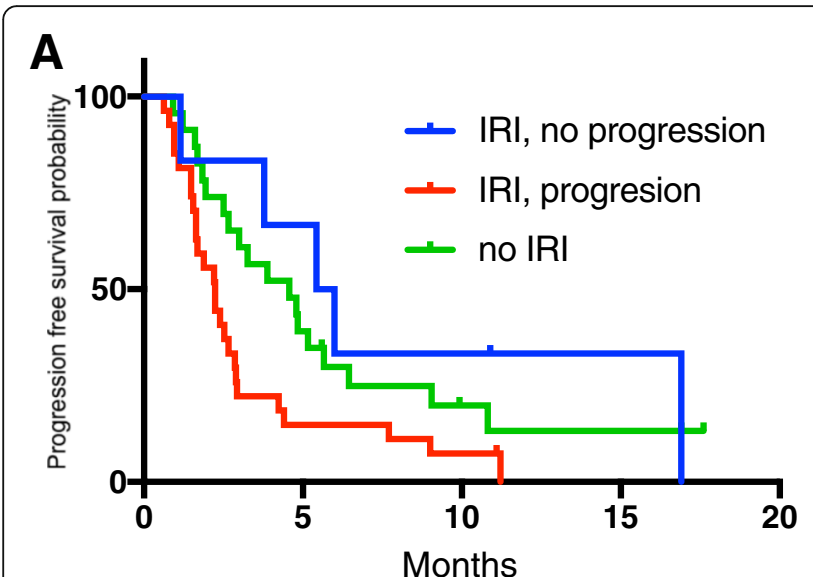

Prior IRI without progression $(n=6)$ : Prior IRI with progression $(n=27)$ : No prior IRI $(n=23)$

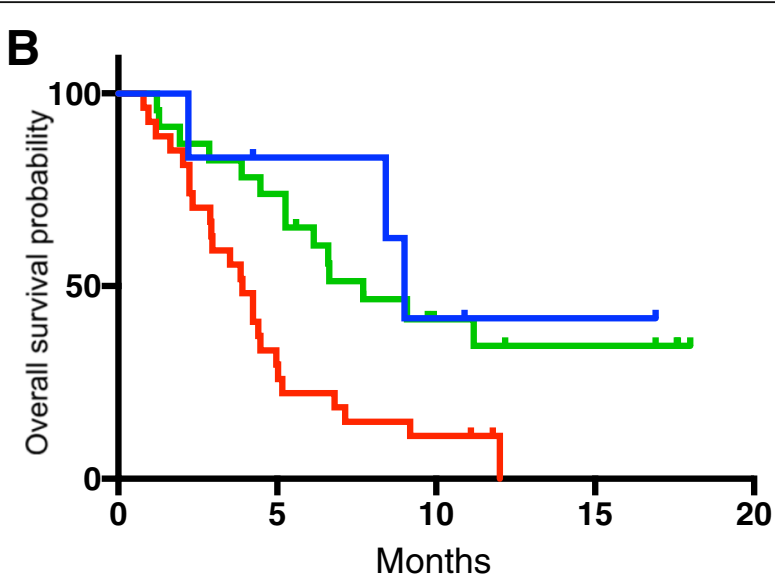

$\begin{array}{ll}\text { mPFS (mo) } & \text { mos (mo) } \\ 5.7 & 9.0 \\ 2.2 & 3.9 \\ 4.6 & 7.7\end{array}$

PFS

IRI, no progression v IRI, progression
no prior IRI v IRI, progression
IRI, no progression v no prior IRI

IRI, no progression v IRI, progression

IRI, no progression v no prior IR
Log-rank test (p) HR (logrank)

$0.041 \quad 0.41(0.19$ to 0.86$)$

$0.022 \quad 0.51(0.28$ to 0.93$)$

$0.55 \quad 0.75$ (0.30 to 1.85$)$

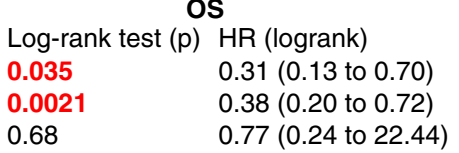

Fig. 1 PFS (a) and OS (b) of patients receiving nal-IRI + 5-FU/LV based on prior irinotecan (IRI) based chemotherapy. Patients were classified based on whether their disease had not progressed on prior IRI-based chemotherapy (IRI, no progression), had progressed on prior IRI-based chemotherapy (IRI, progression), or had not received any prior IRI-based chemotherapy (no-IRI)

\section{Tumor and germline genomics}

Somatic with or without germline genomic results were available for 41 (73\%) patients. The most commonly somatic gene mutations identified in the present study are similar to those identified in previously conducted, large genomic studies, and in similar proportions [9-13]. Activating mutations in KRAS were the most commonly identified, found in $83 \%$ of patients, followed by inactivating mutations in TP53 (66\%), CDKN2A (29\%) and SMAD4 (27\%) (Additional file 1: Table S1). Germline mutations associated with cancer susceptibility were identified in $21 \%$ of patients, a frequency in-line with what our group has recently published in a large patient cohort. [14] BRCA2 was mutated in 3 patients. Although KRAS mutation status was not associated with PFS, TP53 mutation status was associated with significantly shorter PFS (2.2 v $6.0 \mathrm{mo}, p=0.039)$ (Additional file 1: Figure S1). There was a trend to shorter PFS in patients with mutations in SMAD4 and CDKN2A, however, neither of these differences reached statistical significance. Mutations in the four most common somatic genes were not associated with overall survival from the time of advanced disease diagnosis in this cohort. Germline mutations, including those in BRCA1 and BRCA2, were also not associated with differences in PFS or advanced disease overall survival, although the numbers of patients in these cohorts were exceedingly small.

\section{Discussion}

Treatment options for advanced PDAC are expanding but nonetheless finite. Although PDAC remains a challenging disease, the last decade has seen the development of three new and effective combination chemotherapy regimens. The current study is the first report of post-approval, real-world analysis of nal-IRI + 5-FU/LV for the treatment of patients with advanced PDAC. This is also the first study reporting outcomes for patients in an era where two active, combination chemotherapy regimens, FOLFIRINOX and nab-P + Gem, are available for treating patients in the frontline/neoadjuvant settings, and an active, combination chemotherapy regimen, nal-IRI $+5-\mathrm{FU} / \mathrm{LV}$, is available in the second-line setting.

The optimal sequencing of therapy remains undefined, and in practice, is largely defined by patient performance status, age, patient and physician preference. Molecular biomarkers, such as mutations in BRCA $1 / 2$ or microsatellite instability, to guide therapy are found in only a small minority of our patients [15]. For patients receiving FOLFIRINOX in the frontline setting, treatment with nab-P + Gem has been studied in a number of cohort studies. One of the largest was performed by the 

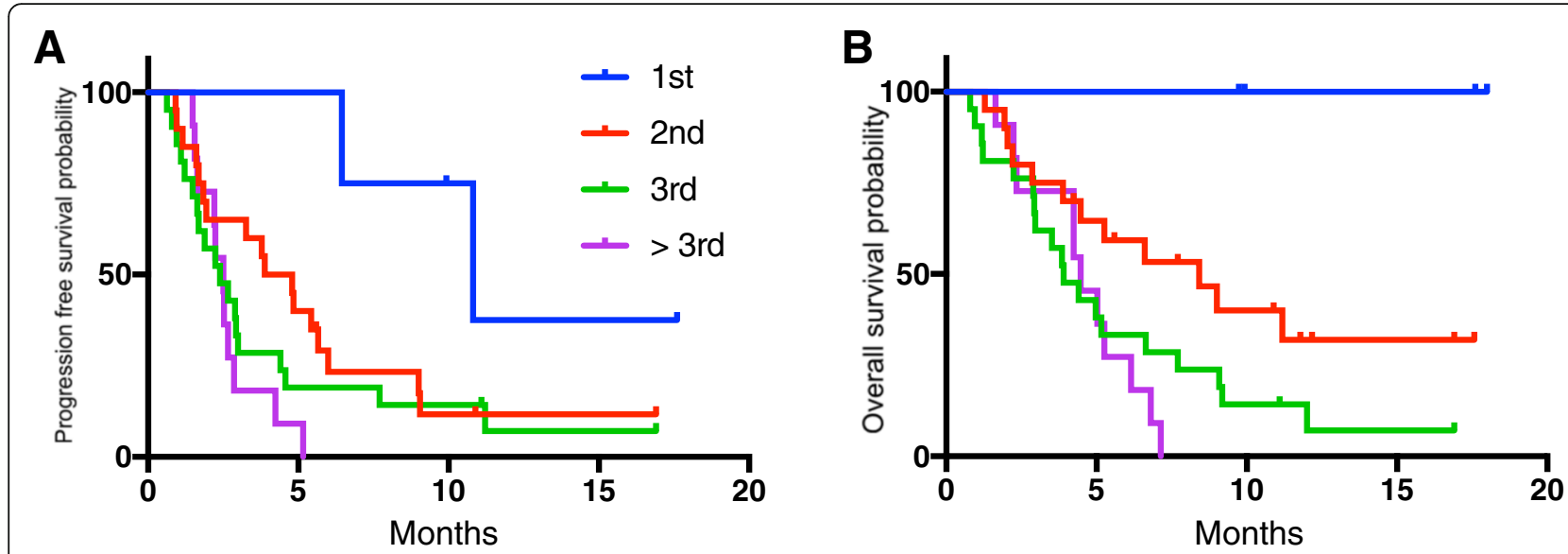

\begin{tabular}{|c|c|c|}
\hline & mPFS (mo) & mOS (mo) \\
\hline $1^{\text {st }}$ line $(n=4)$ : & 10.8 & not reached \\
\hline $2^{\text {nd }}$ line $(n=20)$ : & 4.3 & 8.4 \\
\hline $3^{\text {rd }}$ line $(n=21)$ & 2.4 & 3.9 \\
\hline \multirow[t]{2}{*}{$>3^{\text {rd }}$ line $(n=11)$ : } & 2.5 & 4.5 \\
\hline & PFS & OS \\
\hline Log-rank test for trend & $p=0.0031$ & $p=0.0002$ \\
\hline
\end{tabular}

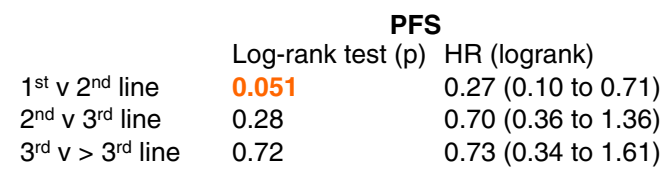

\begin{tabular}{ll}
\multicolumn{2}{c}{ OS } \\
Log-rank test $(p)$ & HR (logrank) \\
0.049 & $0(-1$ to -1$)$ \\
0.059 & $0.51(0.25$ to 1.03$)$ \\
0.46 & $0.76(0.35$ to 1.66$)$
\end{tabular}

Fig. 2 PFS (a) and OS (b) of patients receiving nal-IRI + 5-FU/LV based on line of therapy. Patients were classified based on the line of advanceddisease chemotherapy when nal-IRI + 5-FU/LV was administered

French AGEO (Association des Gastro-Entérologues Oncologues), [16] which studied a similarly sized cohort $(N=57)$ as our current study. Portal and colleagues found reasonable PFS (5.1 mo), OS $(8.8 \mathrm{mo})$ and an encouraging median OS of 18 months from the beginning of advanced disease therapy. For patients receiving nab-P + Gem chemotherapy in the frontline setting, a number of 5-FU-based chemotherapy regimens have been studied. Chiorean and colleagues performed a retrospective analysis of patients enrolled in the pivotal MPACT study to evaluate 2nd therapy received [6]. In patients who received 2nd-line therapy, primarily 5-FU-based, after frontline nab-P + Gem, overall survival was 12.8 mo. The most common 5-FU-based regimens administered contained oxaliplatin. Irinotecan-based chemotherapy was uncommon, and none of these individuals received nal-IRI + 5-FU/LV.

Before the approval of nal-IRI $+5-\mathrm{FU} / \mathrm{LV}$, the most common regimens for treatment after failure of gemcitabine-based chemotherapy were FOLFIRI and FOLFOX. The activity of FOLFIRI has been studied in a number of single arm studies. In one of the largest such studies, Zaniboni and colleagues found PFS and OS of 3.2 and 5 mo, respectively [17]. No randomized studies have been performed to support the efficacy of FOLFIRI in the 2nd line. Two randomized studies investigating the activity of 5-FU and oxaliplatin combinations report conflicting results. The randomized phase III CONKO-003 trial demonstrated a benefit of OFF, a 5 -FU and oxaliplatin regimen commonly administered in Europe, compared to 5-FU alone, with PFS of $2.9 \mathrm{v}$ 2.0 mo $(p=0.019)$, respectively, and OS of $5.9 \mathrm{v} 3.3 \mathrm{mo}$ $(p=0.010)$, respectively [18]. By contrast, the PANCREOX trial demonstrated no benefit of mFOLFOX6 compared with 5-FU, with PFS of 3.1 v $2.9 \mathrm{mo}$, and surprisingly a detriment in OS, 6.1 v $9.9 \mathrm{mo}(p=0.02)$ [19]. A single randomized phase II study has compared second line therapy with FOLFIRI to FOLFOX [20]. Both regimens performed similarly, with PFS of 1.9 and 1.4 mo, respectively, and OS of 3.8 and 3.4 mo, respectively. Overall survival from beginning of frontline therapy was $10.8 \mathrm{mo}$ for both groups. A recent meta-analysis performed by Sonbol and colleagues comparing second-line therapies concluded that although both oxaliplatin and irinotecan improved PFS compared with 5-FU alone, only irinotecan appeared to improve OS [21].

Nal-IRI is a liposomal encapsulated formulation of irinotecan with favorable pharmacokinetic properties as 

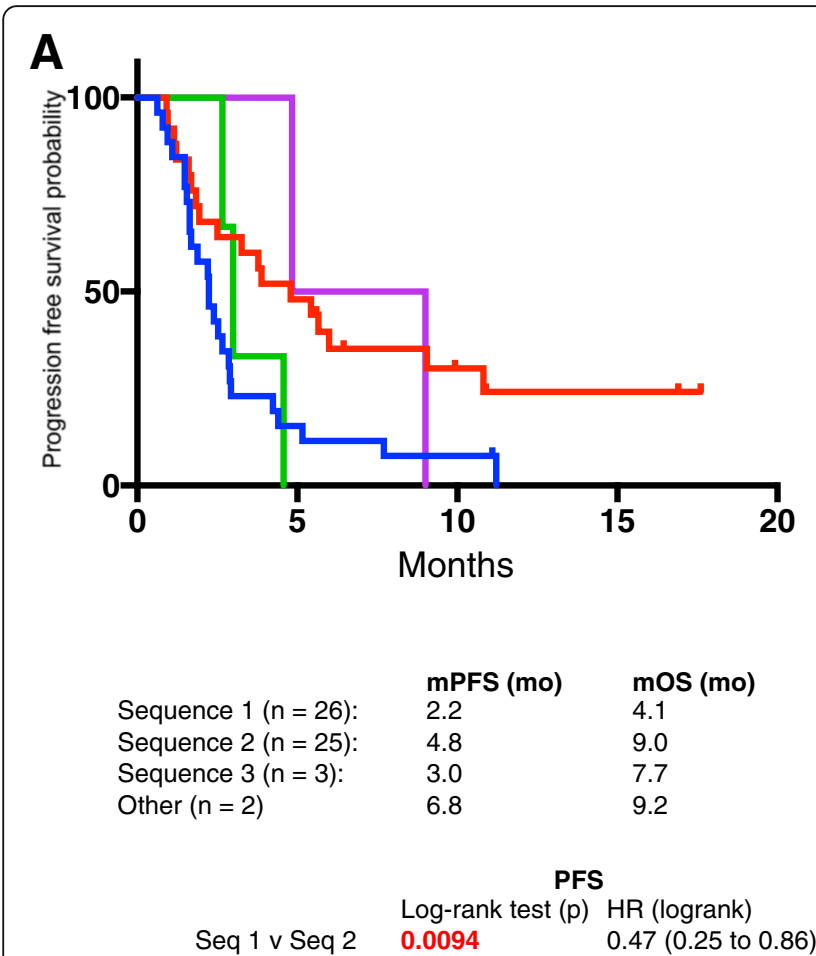

B

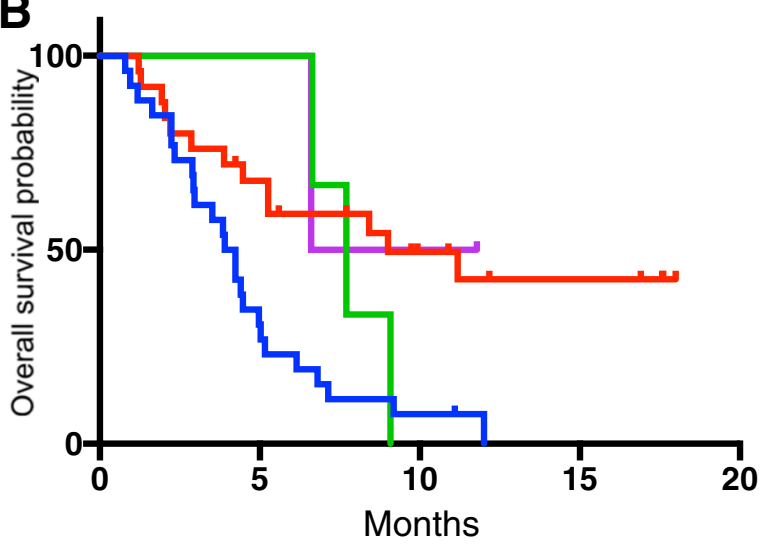

FOLF(IRIN)OX <-> gem/nab -> 5-FU/nal-IRI

+ gem $/$ nab $->$ 5-FU/nal-IRI

gem/nab -> gem/cap -> 5-FU/nal-IRI

+ other

os

Log-rank test (p) HR (logrank)

$0.0006 \quad 0.34(0.18$ to 0.65$)$

Fig. 3 PFS (a) and OS (b) of patients receiving nal-IRI + 5-FU/LV based on treatment sequence. Patients were classified based on common treatment sequences utilized

demonstrated in preclinical [5] and preliminary clinical studies [22]. These results led to a phase II trial, [23] then the randomized phase III NAPOLI-1 trial. [8] NAPOLI-1 was a global study which enrolled 417 patients who previously received Gem-based chemotherapy. Patients were initially randomized to receive either nal-IRI monotherapy dosed at $120 \mathrm{mg} / \mathrm{m}^{2}$ every 3 weeks or 5 -FU/LV monotherapy dosed at $2000 \mathrm{mg} / \mathrm{m}^{2} /$ continuous infusion over $24 \mathrm{~h}$ weekly for 4 out of every

Table 4 Dose reductions and attributed reasons for dose reductions of nal-IRI + 5-FU/LV

\begin{tabular}{ll}
\hline Number of dose reductions & $N=18(\%)$ \\
\hline 1 & $15(83)$ \\
2 & $3(17)$ \\
Reason attributed for dose & \\
Fatigue & $8(44)$ \\
Diarrhea & $8(44)$ \\
Nausea & $2(11)$ \\
Neutropenia & $2(11)$ \\
Anorexia & $2(11)$ \\
Abdominal cramping & $1(6)$ \\
Ageusia & $1(6)$ \\
Not defined & $1(6)$ \\
\hline
\end{tabular}

6-week cycle. A third arm, nal-IRI +5 -FU/LV, dosed at nal-IRI $\left(70 \mathrm{mg} / \mathrm{m}^{2}\right)$ with 5 -FU/LV $\left(2400 \mathrm{mg} / \mathrm{m}^{2} /\right.$ continuous infusion over $46 \mathrm{~h}$ ), was added once the phase II dose of the combination was established. As previously discussed, NAPOLI-1 demonstrated both PFS (3.1 vs 1.5 mo, $p=0.0001$ ) and OS benefit (6.1 vs $4.2 \mathrm{mo}, p=0.012$ ) of nal-IRI + 5-FU/LV compared with 5-FU. In our current study, PFS (2.88 mo) for all patients treated with similar to that seen in the NAPOLI-1 study. A number of key factors were significantly associated with longer survival, including

Table 5 Adverse events and serious (grade 3 or 4) adverse events reported

\begin{tabular}{lll}
\hline & MSKCC & \\
\hline Treatment & nal-IRI +5-FU/LV & \\
Patients & 56 & grade 3/4 (\%) \\
toxicities & any grade (\%) & $2(4)$ \\
Nausea & $33(59)$ & $2(4)$ \\
Vomiting & $18(32)$ & $1(2)$ \\
Diarrhea & $35(63)$ & $1(2)$ \\
Fatigue & $45(80)$ & $0(0)$ \\
Anorexia & $32(57)$ & $1(2)$ \\
Neutropenia & $16(29)$ & $10(18)$ \\
Anemia & $50(89)$ &
\end{tabular}


earlier line of therapy, non-progression on prior irinotecan-based chemotherapy, and dose-reductions while on treatment. In this real-world study, safety was comparable to that seen in the NAPOLI-1 study. The main toxicities seen were fatigue, gastrointestinal toxicities and cytopenias. The incidence of grade 3 or 4 toxicities was low. As part of the NAPOLI-1 study, patients found to be homozygous for the UGT1A1*28 allele were dosed at $50 \mathrm{mg} / \mathrm{m}^{2}$, then dose escalated to $70 \mathrm{mg} / \mathrm{m}^{2}$ in the absence of toxicity. Reassuringly, a separate safety analysis of the NAPOLI-1 study found that patients homozygous for the UGT1A1*28 allele (7/117) experienced similar treatment toxicity compared to those without [24]. Patients treated at our institution are not routinely tested for UGT1A1 genotype. Of note, the median starting dose administered of $55 \mathrm{mg} / \mathrm{m}^{2}$ is below that used in the NAPOLI-1 trial. This pre-emptive dose reduction represents real-world practice patterns and likely played a major role in the low rate of serious adverse events seen. Neither starting dose, nor dose reductions were associated with worse outcomes with regards to PFS or OS. This observation has been made in other regimens used for the treatment of advanced PDAC. For example, Ahn and colleagues found improved safety and promising efficacy when nab-P + Gem was administered at a lower, every other week, frequency [25]. Similarly, FOLFIRINOX with a variety of dose modifications is currently being studied. Two studies have found that dose reductions result in improved safety and similar [26] if not improved [27] efficacy. Lee and colleagues have developed a tool to optimize dose intensity for both toxicity and efficacy and applied their approach to FOLFIRINOX. [28] Studies to systematically examine this and other strategies to improve patient tolerance and outcomes should be undertaken.

With the increased prevalence of tumor somatic and patient germline sequencing, our ability to study the relationship between genomics and treatment response and survival will grow. A number of prior studies have studied gene mutations in KRAS, CDKN2A, TP53 and SMAD4 with regards to survival with mixed results. Hayashi and colleagues found that fewer numbers of mutations in these 4 key genes were associated with better prognosis [29]. Other studies have similarly found low p53 expression, [30] mutations in p16 and TP53, [31] and SMAD4 [32] as predictive of poor prognosis. While mutations in these genes did not correlate with overall survival from advanced stage disease in our patient cohort, there was a correlation between TP53 mutation status and PFS on nal-IRI + 5-FU/LV treatment, with a trend seen for CDKN2A and SMAD4. One preclinical study has previously demonstrated a relationship between TP53 mutation status and irinotecan sensitivity
[33]. While no definitive conclusions can be drawn from a study of this size, our results suggest an interesting pharmacogenomic signal that merits further study and validation in larger, controlled patient cohorts.

The overall survival seen across all sequences of treatment was encouraging. In particular, patients receiving frontline nab-P + Gem followed by 2nd line nal-IRI + 5-FU/LV (Sequence 2) experienced an OS of 23.0 mo from the time of advanced disease diagnosis. Patients treated with FOLFIRINOX and nab-P + Gem prior to nal-IRI + 5-FU/LV (Sequence 1) also experienced excellent OS (25.5 mo), however, this was not significantly longer. This study represents the first published experience documenting survival in a patient population receiving treatment with access to all modern, FDA-approved chemotherapeutic agents. Given the toxicities experienced by some patients receiving FOLFIRINOX, the excellent survival seen in patients who did not receive FOLFIRINOX (Sequence 2) is encouraging and further studies to explore optimal sequencing are warranted. Overall advanced disease survival seen in our study compares favorably to OS reported with sequential nab-P + Gem then 5-FU-based chemotherapy (13.5 mo) [6] and sequential FOLFIRINOX and nab-P + Gem (18 mo). [16] Patient selection is likely a critical issue. Further studies are warranted to confirm the prolonged OS outcomes seen in this study. Patients who received nal-IRI + 5-FU/LV in the frontline metastatic setting experienced prolonged mPFS (10.82 mo) and mOS (not reached), however, the number of patients was very small. The use of nal-IRI for the frontline treatment of patients deserves further evaluation, and an ongoing study (ClinicalTrials.gov Identifier NCT02551991) will hopefully provide a definitive answer to this question.

As a single institution, retrospective analysis, the current study has limitations. Only patients without significant deterioration after prior gemcitabine-based chemotherapy and remained eligible for nal-IRI + 5-FU/LV chemotherapy were included. Patients treated at our tertiary referral center may not experience the same outcomes as patients treated in the community. Nevertheless, our results are encouraging and support continued utilization and study of the nal-IRI + 5-FU/LV regimen to treat patients with advanced pancreatic cancer, and to further optimize selection of patients most likely to benefit.

\section{Conclusions}

This real-world study supports the findings of NAPOLI-1, demonstrating the safety and efficacy of nal-IRI + 5-FU/LV for the treatment of advanced PDAC following gemcitabine-based chemotherapy. Patients receiving nal-IRI + 5-FU/LV in earlier lines of therapy, and without irinotecan-refractory disease, experienced 
significantly longer PFS. Dose reductions were not associated with worse outcomes. Exploratory genetic predictors of response identified candidates which warrant validation. Promising OS was seen integrating nal-IRI + 5-FU/LV sequentially with active combination chemotherapy.

\section{Additional file}

Additional file 1: Figure S1. PFS and tumor genomics. PFS was determined based on presence (mutant) or absence (wild type) of mutations in key tumor suppressor genes associated with PDAC: TP53 (A), SMAD4 (B) and CDKN2A (C). Table S1. Germline and somatic mutations identified using MSK-IMPACT sequencing. (PDF $165 \mathrm{~kb}$ )

\section{Abbreviations}

AE: adverse event; CA 19-9: carbohydrate antigen 19-9; CT: computed tomography; DR: dose reductions; ECOG: Eastern Cooperative Oncology Group; EMR: electronic medical record; FOLFIRINOX: folinic acid, 5-fluorouracil, irinotecan and oxaliplatin; MSKCC: Memorial Sloan Kettering Cancer Center; nab-P + Gem: nab-paclitaxel + gemcitabine; nal-IRI + 5-FU/LV: nanoliposomal irinotecan with fluorouracil/leucovorin; NCI-CTCAE: National Cancer Institute Common Terminology Criteria for Adverse Events; NGS: next generation sequencing: OS: overall survival; PDAC: pancreatic ductal adenocarcinoma; PFS: performance status, progression free survival; SAE: serious adverse event

\section{Acknowledgements}

The study has been presented in part at the 2018 Gastrointestinal Cancers symposium

\section{Funding}

KHY (Research reported in this manuscript was supported by National Cancer Institute of the National Institutes of Health under award number R01CA202762).

\section{Availability of data and materials}

The datasets used and/or analysed during the current study are available from the corresponding author on reasonable request.

\section{Authors' contributions}

DCG and KHY conceived and designed the study, DCG, KHY and EOR analyzed and interpreted the data, DCG, RLP, CMC and KHY acquired data, AMD, GYK, JL, $J \mathrm{JH}, \mathrm{AMV}, \mathrm{EOR}$ and $\mathrm{KHY}$ enrolled patients and collected patient information and data. DCG, RLP, CMC, AMD, GYK, JL, JJH, AMV, EOR and KHY were involved in drafting and revising the manuscript, gave final approval and agree to be accountable for all aspects of the work regarding accuracy or integrity.

\section{Ethics approval and consent to participate}

This retrospective analysis was granted a research waiver by the MSKCC Institutional Review Board. The need for informed consent has been waived by the MSKCC IRB per 45 CFR 46.116 and 45 CFR 164.512

\section{Consent for publication}

Not applicable.

\section{Competing interests}

KHY (consultant or advisory role, Ipsen), EOR (consultant or advisory role, Ipsen).

\section{Publisher's Note}

Springer Nature remains neutral with regard to jurisdictional claims in published maps and institutional affiliations.

Received: 1 March 2018 Accepted: 18 June 2018

Published online: 27 June 2018

\section{References}

1. Siegel RL, Miller KD, Jemal A. Cancer statistics, 2017. CA Cancer J Clin. 2017; 67(1):7-30. https://doi.org/10.3322/caac.21387.
2. Rahib L, Smith BD, Aizenberg R, Rosenzweig AB, Fleshman JM, Matrisian LM Projecting cancer incidence and deaths to 2030: the unexpected burden of thyroid, liver, and pancreas cancers in the United States. Cancer Res. 2014; 74(11):2913-21. https://doi.org/10.1158/0008-5472.CAN-14-0155.

3. Conroy T, Desseigne F, Ychou M, Bouche O, Guimbaud R, Becouarn Y, et al. FOLFIRINOX versus gemcitabine for metastatic pancreatic cancer. N Engl J Med. 2011;364(19):1817-25. https://doi.org/10.1056/NEJMoa1011923.

4. Von Hoff DD, Ervin T, Arena FP, Chiorean EG, Infante J, Moore M, et al. Increased survival in pancreatic cancer with nab-paclitaxel plus gemcitabine. N Engl J Med. 2013;369(18):1691-703. https://doi.org/10.1056/ NEJMoa1304369.

5. Drummond DC, Noble CO, Guo Z, Hong K, Park JW, Kirpotin DB. Development of a highly active nanoliposomal irinotecan using a novel intraliposomal stabilization strategy. Cancer Res. 2006;66(6):3271-7. https:// doi.org/10.1158/0008-5472.CAN-05-4007.

6. Chiorean EG, Von Hoff DD, Tabernero J, El-Maraghi R, Wee Ma W, Reni M, et al. Second-line therapy after nab-paclitaxel plus gemcitabine or after gemcitabine for patients with metastatic pancreatic cancer. Br J Cancer. 2016;115(9):e13. https://doi.org/10.1038/bjc.2016.306.

7. Abrams TA, Meyer G, Meyerhardt JA, Wolpin BM, Schrag D, Fuchs CS. Patterns of chemotherapy use in a U.S.-based cohort of patients with metastatic pancreatic Cancer. Oncologist. 2017;22(8):925-33. https://doi.org/ 10.1634/theoncologist.2016-0447.

8. Wang-Gillam A, Li CP, Bodoky G, Dean A, Shan YS, Jameson G, et al. Nanoliposomal irinotecan with fluorouracil and folinic acid in metastatic pancreatic cancer after previous gemcitabine-based therapy (NAPOLI-1): a global, randomised, open-label, phase 3 trial. Lancet. 2016;387(10018):54557. https://doi.org/10.1016/S0140-6736(15)00986-1.

9. Jones S, Zhang X, Parsons DW, Lin JC, Leary RJ, Angenendt P, et al. Core signaling pathways in human pancreatic cancers revealed by global genomic analyses. Science. 2008;321(5897):1801-6.

10. Yachida S, Jones S, Bozic I, Antal T, Leary R, Fu B, et al. Distant metastasis occurs late during the genetic evolution of pancreatic cancer. Nature. 2010; 467(7319):1114-7

11. Biankin AV, Waddell N, Kassahn KS, Gingras MC, Muthuswamy LB, Johns AL, et al. Pancreatic cancer genomes reveal aberrations in axon guidance pathway genes. Nature. 2012;491(7424):399-405. https://doi.org/10.1038/ nature11547.

12. Waddell N, Pajic M, Patch AM, Chang DK, Kassahn KS, Bailey P, et al. Whole genomes redefine the mutational landscape of pancreatic cancer. Nature. 2015:518(7540):495-501. https://doi.org/10.1038/nature14169.

13. Bailey P, Chang DK, Nones K, Johns AL, Patch AM, Gingras MC, et al. Genomic analyses identify molecular subtypes of pancreatic cancer. Nature. 2016:531(7592):47-52. https://doi.org/10.1038/nature16965.

14. Mandelker D, Zhang L, Kemel Y, Stadler ZK, Joseph V, Zehir A, et al. Mutation detection in patients with advanced Cancer by universal sequencing of Cancer-related genes in tumor and normal DNA vs guideline-based germline testing. JAMA. 2017;318(9):825-35. https://doi.org/ 10.1001/jama.2017.11137.

15. Lowery MA, Jordan EJ, Basturk O, Ptashkin RN, Zehir A, Berger MF, et al. Real-time genomic profiling of pancreatic ductal adenocarcinoma: potential Actionability and correlation with clinical phenotype. Clin Cancer Res. 2017; 23(20):6094-100. https://doi.org/10.1158/1078-0432.CCR-17-0899.

16. Portal A, Pernot S, Tougeron D, Arbaud C, Bidault AT, de la Fouchardiere C, et al. Nab-paclitaxel plus gemcitabine for metastatic pancreatic adenocarcinoma after Folfirinox failure: an AGEO prospective multicentre cohort. Br J Cancer. 2015;113(7):989-95. https://doi.org/10.1038/bjc.2015.328.

17. Zaniboni A, Aitini E, Barni S, Ferrari D, Cascinu S, Catalano V, et al. FOLFIRI as second-line chemotherapy for advanced pancreatic cancer: a GISCAD multicenter phase II study. Cancer Chemother Pharmacol. 2012:69(6):1641-5. https://doi.org/10.1007/s00280-012-1875-1.

18. Oettle H, Riess H, Stieler JM, Heil G, Schwaner I, Seraphin J, et al. Second-line oxaliplatin, folinic acid, and fluorouracil versus folinic acid and fluorouracil alone for gemcitabine-refractory pancreatic cancer: outcomes from the CONKO-003 trial. J Clin Oncol. 2014;32(23):2423-9. https://doi.org/10.1200/ JCO.2013.53.6995.

19. Gill S, Ko YJ, Cripps C, Beaudoin A, Dhesy-Thind S, Zulfiqar M, et al. PANCREOX: a randomized phase III study of fluorouracil/Leucovorin with or without Oxaliplatin for second-line advanced pancreatic Cancer in patients who have received gemcitabine-based chemotherapy. J Clin Oncol. 2016; 34(32):3914-20. https://doi.org/10.1200/JCO.2016.68.5776. 
20. Yoo C, Hwang JY, Kim JE, Kim TW, Lee JS, Park DH, et al. A randomised phase II study of modified FOLFIRI.3 vs modified FOLFOX as second-line therapy in patients with gemcitabine-refractory advanced pancreatic cancer. Br J Cancer. 2009:101(10):1658-63. https://doi.org/10.1038/sj.bjc.6605374.

21. Sonbol MB, Firwana B, Wang Z, Almader-Douglas D, Borad MJ, Makhoul I, et al. Second-line treatment in patients with pancreatic ductal adenocarcinoma: a meta-analysis. Cancer. 2017;123(23):4680-6. https://doi. org/10.1002/cncr.30927.

22. Chang TC, Shiah HS, Yang CH, Yeh KH, Cheng AL, Shen BN, et al. Phase I study of nanoliposomal irinotecan (PEPO2) in advanced solid tumor patients. Cancer Chemother Pharmacol. 2015;75(3):579-86. https://doi.org/ 10.1007/s00280-014-2671-x.

23. Ko AH, Tempero MA, Shan YS, Su WC, Lin YL, Dito E, et al. A multinational phase 2 study of nanoliposomal irinotecan sucrosofate (PEP02, MM-398) for patients with gemcitabine-refractory metastatic pancreatic cancer. $\mathrm{Br}$ J Cancer. 2013;109(4):920-5. https://doi.org/10.1038/bjc.2013.408.

24. Chen LT, Siveke J, Wang-Gillam A, Hubner R, Pant S, Dragovich T, et al. PD023Safety across subgroups in NAPOLI-1: a phase 3 study of nal-IRI (MM398) \pm 5 -fluorouracil and leucovorin (5-FU/LV) versus 5-FU/LV in metastatic pancreatic cancer (mPAC) previously treated with gemcitabine-based therapy. Ann Oncol. 2016;27(Suppl 2):ii1 10-ii. https://doi.org/10.1093/ annonc/mdw200.23.

25. Ahn DH, Krishna K, Blazer M, Reardon J, Wei L, Wu C, et al. A modified regimen of biweekly gemcitabine and nab-paclitaxel in patients with metastatic pancreatic cancer is both tolerable and effective: a retrospective analysis. Ther Adv Med Oncol. 2017;9(2):75-82. https://doi.org/10.1177/ 1758834016676011.

26. Li X, Ma T, Zhang Q, Chen YG, Guo CX, Shen YN, et al. Modified-FOLFIRINOX in metastatic pancreatic cancer: a prospective study in Chinese population. Cancer Lett. 2017;406:22-6. https://doi.org/10.1016/j.canlet.2017.07.012.

27. Ohba A, Ueno H, Sakamoto Y, Kondo S, Morizane C, Okusaka T. Retrospective comparison of modified FOLFIRINOX with full-dose FOLFIRINOX for advanced pancreatic cancer: A Japanese cancer center experience. J Clin Oncol. 2018;36(4_suppl):469. https://doi.org/10.1200/JCO. 2018.36.4_suppl.469.

28. Lee JC, Kim JW, Ahn S, Kim HW, Lee J, Kim YH, et al. Optimal dose reduction of FOLFIRINOX for preserving tumour response in advanced pancreatic cancer: using cumulative relative dose intensity. Eur I Cancer. 2017;76:125-33. https://doi.org/10.1016/j.ejca.2017.02.010.

29. Hayashi H, Kohno T, Ueno H, Hiraoka N, Kondo S, Saito M, et al. Utility of assessing the number of mutated KRAS, CDKN2A, TP53, and SMAD4 genes using a targeted deep sequencing assay as a prognostic biomarker for pancreatic Cancer. Pancreas. 2017;46(3):335-40. https://doi.org/10.1097/MPA. 0000000000000760 .

30. Grochola LF, Taubert H, Greither T, Bhanot U, Udelnow A, Wurl P. Elevated transcript levels from the MDM2 P1 promoter and low p53 transcript levels are associated with poor prognosis in human pancreatic ductal adenocarcinoma. Pancreas. 2011:40(2):265-70.

31. Luo Y, Tian L, Feng Y, Yi M, Chen X, Huang Q. The predictive role of $p 16$ deletion, p53 deletion, and polysomy 9 and 17 in pancreatic ductal adenocarcinoma. Pathol Oncol Res. 2013;19(1):35-40. https://doi.org/10. 1007/s12253-012-9555-3.

32. Blackford A, Serrano OK, Wolfgang CL, Parmigiani G, Jones S, Zhang X, et al. SMAD4 gene mutations are associated with poor prognosis in pancreatic cancer. Clin Cancer Res. 2009;15(14):4674-9. https://doi.org/10.1158/10780432.CCR-09-0227.

33. Abal M, Bras-Goncalves R, Judde JG, Fsihi H, De Cremoux P, Louvard D, et al. Enhanced sensitivity to irinotecan by Cdk1 inhibition in the p53-deficient HT29 human colon cancer cell line. Oncogene. 2004;23(9):1737-44. https:// doi.org/10.1038/sj.onc.1207299.

Ready to submit your research? Choose BMC and benefit from:

- fast, convenient online submission

- thorough peer review by experienced researchers in your field

- rapid publication on acceptance

- support for research data, including large and complex data types

- gold Open Access which fosters wider collaboration and increased citations

- maximum visibility for your research: over $100 \mathrm{M}$ website views per year

At BMC, research is always in progress.

Learn more biomedcentral.com/submissions 